\title{
NOTE ON QUARTILES AND ALLIED MEASURES*
}

BY DUNHAM JACKSON

If a number of values $a_{1}, a_{2}, \cdots, a_{n}$ of a quantity $x$ have been observed, the lower quartile of this set of observations may be roughly described as a number $x_{1}$ such that one fourth of the $a$ 's are less than $x_{1}$ and three fourths of them are greater than $x_{1}$. Something more is needed for an exact definition, inasmuch as the condition stated either leads to an indeterminate value or is impossible of realization, according to circumstances. If $x_{1}$ is defined as a value of $x$ which reduces to a minimum the expression

$$
S_{1}=\sum_{i=1}^{n} \varphi_{1}\left(x-a_{i}\right)
$$

where $\varphi_{1}(x)=\frac{3}{4} x$ for $x \geqq 0, \varphi_{1}(x)=-\frac{1}{4} x$ for $x \leqq 0$, there will always be at least one value of $x_{1}$ satisfying the condition, and this will agree with the value of the quartile as ordinarily understood, but if $n=4 k$ and $a_{k} \neq a_{k+1}$, when the $a$ 's are arranged in order of increasing algebraic magnitude, any number between $a_{k}$ and $a_{k+1}$ will meet the requirement. It is the purpose of this note to show that a unique determination results in all cases from a definition analogous to one which the author recently suggested for the median. $\dagger$ As in the previous instance, the definition is admittedly of theoretical rather than practical interest. The discussion is put in such a form as to apply equally well to an arbitrary percentile or other measure of similar character, the ratio $1: 3$ being replaced by any other positive ratio.

Let $a_{1}, \cdots, a_{n}$ be a set of real numbers (not necessarily all distinct) arranged in ascending order of magnitude algebraically, and let $c$ be an arbitrary number of the interval $0<c$ $<1$. For $p \geqq 1$, let a function $\varphi_{p}(x)$ be defined as follows: $\varphi_{p}(x)=(1-c) x^{p}$ for $x \geqq 0, \quad \varphi_{p}(x)=c(-x)^{p}$ for $x \leqq 0$.

* Presented to the Society, October 28, 1922.

$\dagger$ Note on the median of a set of numbers, this BuLletin, vol. 27 (1920-21), pp. 160-164. 
The value of $c$ will be kept constant throughout, and need not be indicated in the notation for the function $\varphi$. Let

$$
S_{p}=\sum_{i=1}^{n} \varphi_{p}\left(x-a_{i}\right)
$$

and let $x_{p}$ stand for the value of $x$, or any value of $x$, which reduces $S_{p}$ to a minimum. It is to be shown that $x_{p}$ is uniquely determined for each value of $p>1$; that as $p$ approaches 1 , $x_{p}$ approaches $x_{1}$, if $x_{1}$ has a determinate value; and that in the contrary case, $x_{p}$ approaches a definite limit belonging to the interval within which $x_{1}$ is indeterminate.

Consider first the case that $c n$ is not an integer. Let $k$ be the integer such that $k-1<c n<k$. Then $x_{1}$ definitely has the value $a_{k}$. For as $x$ changes from $a_{k}$ to $a_{k}+\delta$, at least $k$ terms of the sum $S_{1}$ are increased, each by the amount $(1-c) \delta$, and not more than $n-k$ terms are diminished, the amount of decrease in each case being $c \delta$ at most, so that the net change in $S_{1}$ is at least

$$
k(1-c) \delta-(n-k) c \delta=(k-c n) \delta>0 ;
$$

and as $x$ changes from $a_{k}$ to $a_{k}-\delta$, at least $n-k+1$ terms are increased, not more than $k-1$ are diminished, and the net change is at least

$$
(n-k+1) c \delta-(k-1)(1-c) \delta=(c n-k+1) \delta>0 .
$$

It will be shown that $x_{p}$ is uniquely determined for each value of $p>1$, and that $\lim _{p=1} x_{p}=a_{k}$.

When $p>1$, the function $\varphi_{p}(x)$ is continuous and has a sontinuous derivative for all values of $x$, including $x=0$. Since $S_{p}(x)$ is continuous and becomes infinite as $x$ becomes infinite in either direction, it must have at least one minimum. A necessary condition for a minimum is the vanishing of $S_{p}{ }^{\prime}(x)$. But it is readily seen, either by inspection or by writing down the explicit formulas, that $\varphi_{p}^{\prime}(x)$ always increases when $x$ increases, so that $S_{p}{ }^{\prime}(x)$ likewise increases when $x$ increases, and can vanish only once. This proves the existence and uniqueness of $x_{p}$.

Let $\epsilon$ be an arbitrarily small positive quantity, and let $r$ be an index such that $a_{r}<a_{k}+\epsilon \leqq a_{r+1}$; it is clear that $r \geqq k$. 
It follows from the definition of $\varphi$ that $\varphi_{p}^{\prime}(x)=(1-c) p x^{p-1}$ or $-c p(-x)^{p-1}$, according to the sign of $x$, and hence

$$
\begin{aligned}
& \frac{1}{p} S_{p}{ }^{\prime}\left(a_{k}+\epsilon\right)=(1-c)\left(a_{k}+\epsilon-a_{1}\right)^{p-1}+\cdots \\
& +(1-c)\left(a_{k}+\epsilon-a_{r}\right)^{p-1}-c\left(a_{r+1}-a_{k}-\epsilon\right)^{p-1}-\cdots \\
& \quad-c\left(a_{n}-a_{k}-\epsilon\right)^{p-1} .
\end{aligned}
$$

When $p$ approaches 1 , each of the first $r$ terms on the right, apart from the factor $1-c$, approaches the limit 1, and each of the remaining terms, apart from the factor $c$, but inclusive of the algebraic sign, approaches -1 or possibly 0 . So

$\lim _{p=1} S_{p}^{\prime}\left(a_{k}+\epsilon\right) \geqq r(1-c)-(n-r) c=r-c n \geqq k-c n>0$.

Similarly,

$$
\lim _{p=1} S_{p}{ }^{\prime}\left(a_{k}-\epsilon\right)<0 .
$$

For if the definition of $r$ is changed so that $a_{r} \leqq a_{k}-\epsilon<a_{r+1}$, then $r \leqq k-1$, and

$$
\begin{aligned}
& \frac{1}{p} S_{p}{ }^{\prime}\left(a_{k}-\epsilon\right)=(1-c)\left(a_{k}-\epsilon-a_{1}\right)^{p-1}+\cdots \\
& +(1-c)\left(a_{k}-\epsilon-a_{r}\right)^{p-1}-c\left(a_{r+1}-a_{k}+\epsilon\right)^{p-1}-\cdots \\
& \quad-c\left(a_{n}-a_{k}+\epsilon\right)^{p-1}, \\
& \begin{aligned}
\lim _{p=1} S_{p}{ }^{\prime}\left(a_{k}-\epsilon\right) \leqq r(1-c)-(n-r) c & =r-c n \\
& \leqq k-1-c n<0 .
\end{aligned}
\end{aligned}
$$

So the value $x_{p}$ for which $S_{p}{ }^{\prime}$ vanishes must be between $a_{k}-\epsilon$ and $a_{k}+\epsilon$ when $p$ is sufficiently near 1 , or, in other words, $\lim _{p=1} x_{p}=a_{k}$.

Suppose now that $c n$ is an integer, and let $c n=k$. If it happens that $a_{k}=a_{k+1}$, reasoning similar to that presented above shows that $x_{1}=a_{k}$ and $\lim _{p=1} x_{p}=a_{k}$, as before.

This special case being left aside, it is to be assumed that $a_{k}<a_{k+1}$. The definition of $x_{1}$ is satisfied by $a_{k}$ or $a_{k+1}$ or any intermediate value. For $p>1$, on the other hand, $x_{p}$ is seen to be uniquely determined, by the same argument as was used before. Furthermore, it is recognized that

$$
\begin{aligned}
& \lim _{p=1} S_{p}{ }^{\prime}\left(a_{k}\right)=(k-1)(1-c)-(n-k) c \\
& =k-1+c-c n=c-1<0, \\
& \lim _{p=1} S_{p}{ }^{\prime}\left(a_{k+1}\right)=k(1-c)-(n-k-1) c=k-c n+c=c>0, \\
& \text { so that } a_{k}<x_{p}<a_{k+1} \text { when } p \text { is sufficiently near } 1 \text {. It }
\end{aligned}
$$


remains to be shown that $x_{p}$ approaches a definite limit as $p$ approaches 1 .

Let $x$ have a value between $a_{k}$ and $a_{k+1}$. For $i=1,2, \cdots, k$, $x-a_{i}$ is positive, and

$$
\begin{aligned}
& \left(x-a_{i}\right)^{p-1}=e^{(p-1) \log \left(x-a_{i}\right)} \\
& \quad=1+(p-1) \log \left(x-a_{i}\right)+\frac{1}{2}(p-1)^{2} \log ^{2}\left(x-a_{i}\right)+\cdots \\
& \quad=1+(p-1)^{\prime} \log \left(x-a_{i}\right)+(p-1)^{2} \rho_{i}(x, p),
\end{aligned}
$$

where $\rho_{i}(x, p)$ is a function which approaches $\frac{1}{2} \log ^{2}\left(x-a_{\imath}\right)$, and so remains finite, if $x$ is held fast and $p$ approaches 1 . For $i>k$,

$$
\left(a_{i}-x\right)^{p-1}=1+(p-1) \log \left(a_{i}-x\right)+(p-1)^{2} \rho_{i}(x, p),
$$

where $\rho_{i}(x, p)$ again remains finite for fixed $x$ as $p$ approaches 1 . If these values are substituted in the explicit expression for $(1 / p) S_{p}{ }^{\prime}(x)$, there will be $k$ terms each equal to $(1-c)$ and $(n-k)$ terms each equal to $(-c)$, which will destroy each other, because of the relation $c n=k$, and each of the remaining terms will have a factor $p-1$, so that we may write

$$
\begin{aligned}
& \frac{1}{p(p-1)} S_{p}{ }^{\prime}(x)=(1-c)\left[\log \left(x-a_{1}\right)+\cdots+\log \left(x-a_{k}\right)\right] \\
& \quad-c\left[\log \left(a_{k+1}-x\right)+\cdots+\log \left(a_{n}-x\right)\right]+(p-1) \rho(x, p) \\
& \quad=\log \frac{\left[\left(x-a_{1}\right) \cdots\left(x-a_{k}\right)\right]^{1-c}}{\left[\left(a_{k+1}-x\right) \cdots\left(a_{n}-x\right)\right]^{c}}+(p-1) \rho(x, p),
\end{aligned}
$$

the function $\rho$ remaining finite as $p$ approaches 1 .

As the exponents $c$ and $1-c$ are both positive, the fraction on the right increases steadily from 0 to $+\infty$ as $x$ goes from $a_{k}$ to $a_{k+1}$, and the logarithm increases steadily from $-\infty$ to $+\infty$, taking on the value 0 just once, say for $x=X$. For $x=X+\epsilon$, the logarithm is positive and independent of $p$, while the term $(p-1) \rho(X+\epsilon, p)$ approaches zero as $p$ approaches 1 . So the value of the whole expression on the right is positive when $p-1$ is sufficiently small. For a similar reason, the expression is negative for $x=X-\epsilon$, if $p$ is sufficiently close to 1 . This means that the root of $S_{p}{ }^{\prime}(x)$ is between $X-\epsilon$ and $X+\epsilon$ when $p-1$ is sufficiently small, that is,

$$
\lim _{p=1} x_{p}=X .
$$

The University of Minnesota 